\title{
Race, Discharge Disposition, and Readmissions After Elective Hip Replacement: Analysis of a Large Regional Dataset
}

\author{
Bella Mehta, 2,* Jasvinder A. Singh, Kaylee Ho, ${ }^{4,5}$ Michael Parks, ${ }^{6}$ Charles Nelson, Debra D’Angelo, 4,5 and Said A. Ibrahim ${ }^{4,8}$
}

\begin{abstract}
Purpose: Total hip arthroplasty (THA) is one of the fastest growing procedures. There is increasing evidence that social determinants of health influence health care utilization and outcomes after THA, including postoperative care. We sought to examine how race impacts discharge destination after elective THA, and we assessed the impact of discharge destination on 90-day readmission to an acute care hospital.

Methods: We conducted a retrospective study using data from the Pennsylvania Health Care Cost Containment Council Database. We included patients of African American (AA) or white race undergoing THA, discharge disposition (inpatient rehabilitation facility [IRF], skilled nursing facility [SNF], home health care (HHC), home), and 90-day readmission rates.

Results: Our study included 93,493 primary elective THAs. Compared with whites, AAs were more likely to be discharged to an IRF or SNF or HHC than home after THA. In all age groups, discharge to an IRF, SNF, or HHC for postop care/rehab was associated with higher odds of 90-day readmission as compared with home.

Conclusion: AA race was associated with higher odds of discharge to an institution (IRF/SNF) or HHC for postTHA care. Disposition to these were associated with significantly higher risk of 90-day readmission to acute care hospital compared with home.
\end{abstract}

Keywords: race; rehabilitation; THA; readmissions

\section{Introduction}

Osteoarthritis $(\mathrm{OA})$ is the most prevalent joint disease and a leading source of chronic pain and disability in the United States. ${ }^{1}$ Its prevalence has doubled even after controlling for many factors, including longevity and body mass index (BMI). ${ }^{2}$ Total hip arthroplasty (THA) is currently the only definitive treatment for patients with end-stage hip OA who have failed conservative treatment. THA has successful outcomes and a timely procedure can prevent functional disability in many patients, including the elderly. Elective THA is one of the fastest growing procedures and is the fourth most common surgical procedure performed surgical procedures in the United States. ${ }^{3}$

The utilization of THA has increased exponentially worldwide over the past decade and especially in United States where the rate exceeds $200 / 100,000$ population. ${ }^{4}$ This utilization has also increased in the younger age group. ${ }^{5}$ Its utilization is anticipated to grow and by 2030, the demand for THA is expected to grow up to 572,000 THAs per year. ${ }^{6}$ In addition, there is growing evidence that social determinants of health, such as race, impact THA utilization and its outcomes. Even though the prevalence of Hip OA in

Departments of ${ }^{1}$ Rheumatology and ${ }^{6}$ Adult Reconstruction \& Joint Replacement Service, Hospital for Special Surgery, New York, New York.

Departments of ${ }^{2}$ Rheumatology and ${ }^{4}$ Healthcare Policy and Research, ${ }^{5}$ Division of Biostatistics and Epidemiology, and ${ }^{8}$ Healthcare Delivery Science \& Innovation, Weill Cornell Medicine, New York, New York

${ }^{3}$ Department of Medicine and Division of Epidemiology, University of Alabama at Birmingham, Birmingham, Alabama.

${ }^{7}$ Department of Orthopedic Surgery, Hospital of the University of Pennsylvania, Philadelphia, Pennsylvania.

${ }^{*}$ Address correspondence to: Bella Mehta, MBBS, MS, Hospital for Special Surgery, 535 E 70th Street, New York, NY 10021, E-mail: drbellamehta@gmail.com

(c) Bella Mehta et al. 2019; Published by Mary Ann Liebert, Inc. This Open Access article is distributed under the terms of the Creative Commons License (http://creativecommons.org/licenses/by/4.0), which permits unrestricted use, distribution, and reproduction in any medium, provided the original work is properly cited. 
African Americans (AAs) and whites is similar, AAs have lower utilization of THA. ${ }^{7,8}$

With regard to the outcomes, AAs have worse outcomes as compared with whites with a higher mortality, disability score, and longer length of stay. ${ }^{9-11}$ The rationale for these disparities are complex and intertwined between patient-level, provider-level, systemlevel, and policy-level factors. Utilization of postoperative care and its relationship to outcomes like readmissions is a lesser examined aspect in these disparities. We previously examined this in THA patients between the years 2002-2012 and found that race was a significant predictor of postoperative discharge destination. In addition, the discharge to an institution (inpatient rehabilitation facility [IRF] or skilled nursing facility [SNF]) was associated with increased odds of 90-day readmission to an acute care hospital. ${ }^{12}$

Since 2012, CMS has made several policy innovations to have an affordable and accessible health care system that makes patient outcomes a priority. Medicare Bundled Payment for Care Improvement (BPCI) initiative motivates and incentivize health care systems and providers to administer services efficiently and to better coordinate care, including discharge destination following THA. The hospitals are accountable for costs related to a THA procedure as well as costs associated within 90 days after discharge related to it. ${ }^{13}$ It is not known if these policy innovations had an impact on racial inequities in discharge disposition after THA. Therefore, using more recent data, we sought to examine the racial differences in discharge destination and if this is in turn associated with 90-day readmission to acute care hospital.

\section{Methods}

\section{Data source}

We retrospectively analyzed the Pennsylvania Health Care Cost Containment Council (PHC4) Database (2012-2016), which includes demographic data from all patient discharges from 170 nongovernmental acute care hospitals in the State of Pennsylvania. This includes all hospitals other than Veterans Administration or Military hospitals. The database was used to identify patients who underwent elective primary THA. Patients were identified using the International Classification of Diseases, Ninth Revision, Clinical Modification (ICD-9-CM) code 81.51 for primary THA from 2012 to September 2015, and ICD-10 codes 0SR90xx or OSRB0xx thereafter. This study cohort and methodology has previously been described in detail. ${ }^{14}$
Our primary exposure of interest was race and thus only adults who identified as AAs or white and had an elective primary THA were included in this study. Study exclusion criteria included the following: patients with bilateral hip replacement, age $<18$, unknown race or insurance status, death on the same day of THA surgery or during hospitalization, transfer to a different acute care hospital or to a nonstudy destination (discharge destination other than the ones defined below), hip revision during the same hospitalization, or have more than two prior hip replacements (likely administrative dataset error).

\section{Outcome measures}

The primary study outcome of interest was discharge disposition following a primary elective THA surgery. The discharge destination categories include IRF, SNF, home with home health care (HHC), and home with routine self-care (Home). In all analyses, discharge to home was used as the reference category. We also examined odds of 90 -day readmissions to acute care hospital as our secondary outcome of interest.

\section{Analytical sample and covariates}

We extracted information on important covariates, such as age, gender, insurance status (Commercial, Medicaid, Medicare, or other government-sponsored health insurance program), 90-day mortality, and clinical- and facility-level variables, from out dataset. We incorporated two facility-level variables related to the characteristics of the hospital at which the patients underwent THA. We used the 2013 U.S. Department of Agriculture's Rural/Urban Continuum Codes to assign the Metro area status to each hospital. ${ }^{15}$ The hospital THA procedure volume was categorized into three ordinal groups based on the volume: $<100,100-199$, and $\geq 200$ THA procedures per year. Complications, including postoperative myocardial infarction, prosthetic device complication, surgical wound infection, and venous thromboembolism, were identified using ICD-9/ 10 codes (Appendix Table A1). Lastly, medical comorbidities were identified using the Quan adaptation of the Elixhauser Comorbidity Index. ${ }^{16,17}$

For our secondary analysis, the primary predictor for 90-day readmission is the type of postsurgical rehabilitation care destinations (IRF, SNF, HHC, and home). The same covariates from the primary analysis were included in the multivariable logistic models for 90-day readmission. The study methods and results are 
described in accordance with the Strengthening of Reporting in Observational Studies in Epidemiology (STROBE) guideline for cohort studies.

\section{Statistical analyses}

We compared the patient-level and facility-level characteristics, along with clinical outcomes, by patient race and stratified it by age group. We tested the associations between race and the various patient-level, facility-level, and outcome variables using Wald chisquare from unadjusted binary or multinomial logistic regression models, accounting for the clustering by Pennsylvania Area Facility. All models for the primary outcome considered race as the independent variable and accounted for clustering by hospital facility. Using similar strategies, patient-level characteristics, facility-level characteristics, and 90-day hospital readmission were compared by postsurgical discharge disposition (considered the independent variable).

We used multinomial logistic generalized linear mixed model ${ }^{18}$ to estimate the unadjusted and adjusted relative risk ratios (aRRRs) of being discharged to IRF, to a SNF, or to HHC (vs. home) after THA in AAs compared with whites. Multivariable models were adjusted for sex, age, insurance type, metro area, volume of cases, surgical complications (postoperative myocardial infarction, prosthetic device complication, surgical wound infection, venous thromboembolism) and Elixhauser Index. Covariates selection is based on clinical and prior knowledge from the scientific literature. In all models, patients were stratified by age group ( $<65$ and 65 years or older). The age-based stratification was performed to account for differences in Medicare eligibility.

The relationship between 90-day readmission and discharge destination was assessed using binary logistic regression, accounting for clustering by hospital facility. Unadjusted and adjusted odds ratios (aORs) of hospital readmission at 90 days were estimated. Multivariable models were adjusted for the same patientlevel and facility-level variables from the primary outcome analysis.

Furthermore, we utilized the coarsened exact matching (CEM) method in assessing the 90 -day readmission outcome to reduce imbalance of covariates between different discharge destinations (IRF, SNF, HHC, and Home). CEM is a nonparametric and nonmodelbased matching method of the class monotonic imbalance bounding ${ }^{19}$ that ensures the balance of covariates between groups before estimation, allowing for less model dependence and reduced statistical bias than without matching. ${ }^{20}$ In CEM, adjusting imbalance in the empirical distribution in one covariate does not affect any other covariates chosen for balancing, which makes CEM more advantageous over other matching methods (King G, Nielsen R, Coberley C, et al. Comparative effectiveness of matching methods for causal inference. Unpublished manuscript. 2011;15:41). CEM was conducted using the CEM package in the 64-bit version of R.3.5.1. ${ }^{21}$ The dataset was matched across the following variables: age, gender, insurance, and 30 clinical comorbidities defined by Quan adaptation of Elixhauser comorbidities. ${ }^{17}$ After matching, 90-day readmission was regressed on discharge disposition using a binary logistic regression model adjusting for the same covariates in prior multivariable logistic regression.

Data management and analyses were conducted using SAS version 9.4 (SAS Institute, Cary, NC) and R.3.5.1 in RStudio (version 1.1.463; RStudio, Inc., Boston, MA).

\section{Results}

Study sample characteristics

Around 99,171 cases of THA were identified between 2012 and 2016 that met our inclusion criteria. We excluded 5678 cases for various reasons (Fig. 1). The final analytic sample was 93,493 .

Baseline demographic and clinical characteristics of the cohort stratified by age group are summarized in Table 1 . Briefly, among the THA surgery patients analyzed, 87,616 patients were white and 6626 were AA. In the age group less than 65 years $(n=44,647), 9.5 \%$ were AA. Around $46.1 \%$ of AA and $47 \%$ of whites in that age group were female; $27 \%$ of AAs and $7.03 \%$ of whites relied on Medicaid insurance; whereas $80.3 \%$ whites and $50.4 \%$ AA relied on commercial insurance. In the age group 65 years and older $(n=48,846), 4.5 \%$ were AA and rest white. Around $61.1 \%$ of whites and $65.5 \%$ of AAs in that age group were female; $0.14 \%$ of whites and $1.53 \%$ of AAs relied on Medicaid insurance; similar proportion of AAs and whites relied on commercial insurance in this older age group $(\sim 12 \%)$.

\section{Characteristics by discharge destination: $<65$ years versus $\geq 65$ years}

Demographic and clinical characteristics by discharge destination were described (Table 2). Briefly, 38.2\% of patients were discharged to either a SNF or an IRF, whereas the others were discharged to home self-care (Home) or HHC. Among AAs, 20.7\%, 38.6\%, 15.6\%, 


\section{Initial sample: $\mathbf{n}=99,171$ \\ All hip replacement records performed from 2012 Q1 through 2016 Q4 (1/1/2012, $12 / 31 / 2016$ )}

1. Race missing or not listed as African American or white $(n=3,526)$

2. More than two previous hip replacements $(n=8)$

3. Went to a non-study discharge destination $(n=1,029)$

4. Age $<18$ years $(n=54)$

5. Missing primary insurance status $(n=28)$

6. Had hip replacement and/or revision during same hospitalization $(n=104)$

7. Died on date of discharge $(n=1)$

8. Bilateral hip replacements $(n=928)$

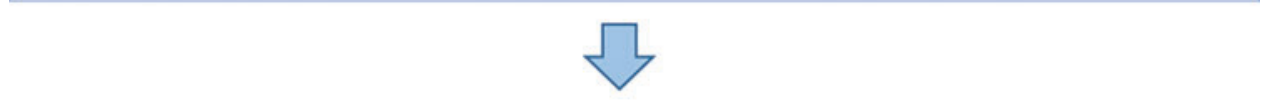

Study Population: $n=93,493$

All qualified hip replacement records performed from 2012 Q1 through 2016 Q4 (1/1/2012, 12/31/2016)

FIG. 1. Sample flow chart and cohort selection.

and $25.1 \%$ were discharged to Home, HHC, SNF, and IRF, respectively. Among women, 22\%, 38\%, 14.7\%, and $25.1 \%$ were discharged to Home, HHC, SNF, and IRF, respectively.

Among those with Medicare, $18.7 \%$ were discharged to home and $41.5 \%$ and $37.5 \%$ were discharged to SNF or IRF, respectively. Thus, a higher percentage of patients were discharged to an institution when patients had Medicare. Among those with commercial insurance, $32.9 \%$ were discharged to home and $34.2 \%$ were discharged to SNF or IRF, showing that a higher proportion was discharged home when they had commercial insurance.

\section{Race and discharge destination}

We estimated relative risk ratios of discharge destination by patient race, adjusting for important patientand facility-level confounders (Fig. 2). Among patients younger than 65 years, compared with whites, AAs were more likely to be discharged to IRF (aRRR: 1.21, 95\% confidence interval [CI]: 1.13-1.29), SNF (aRRR: 1.19, 95\% CI: $1.10-1.29$ ) or HHC (aRRR: $1.17,95 \%$
CI: 1.10-1.25), but not home. Similarly, in the older age group $>65$, compared with whites, AAs were more likely to be discharged to an IRF (aRRR: 1.16, 95\% CI: 1.03-1.31), SNF (aRRR: 1.17, 95\% CI: 1.02-1.32), or HHC (aRRR: 1.16, 95\% CI: 1.04-1.30) compared with Home.

\section{Discharge disposition and 90-day readmission to acute-care hospital}

The association of discharge disposition with 90-day readmission to an acute care hospital was analyzed after adjusting for confounders as well as after CEM (Fig. 3). In the younger age group (less than 65 years) compared with Home, discharge to IRF, SNF, and HHC was associated with higher odds of 90-day readmission to acute care hospital (aOR: 1.47, 95\% CI: 1.32-1.63; aOR: 1.40, 95\% CI: 1.24-1.58; and aOR: 1.48, 95\% CI: $1.34-1.64$, respectively). This association remained statistically significant $(p<0.05)$ after CEM for IRF, SNF, and HHC (aOR: $1.60,95 \%$ CI: 1.39-1.85; aOR: 1.51, 95\% CI: 1.28-1.78; and aOR: 1.61 , 95\% CI: $1.42-1.84$, respectively) compared with Home. 
Table 1. Baseline Characteristics and Outcomes by Race and Age Group $(N=93,493)$

\begin{tabular}{|c|c|c|c|c|c|c|}
\hline \multirow[b]{2}{*}{ Variable, $n(\%)$} & \multicolumn{3}{|c|}{ Years $<65(n=44,647)$} & \multicolumn{3}{|c|}{ Years $\geq 65(n=48,846)$} \\
\hline & WH $(n=40,394)$ & AA $(n=4253)$ & $p^{\mathrm{a}}$ & WH $(n=46,619)$ & $\mathrm{AA}(n=2227)$ & $p$ \\
\hline \multicolumn{7}{|l|}{ Sex } \\
\hline Female & $18,977(47.0)$ & $1960(46.1)$ & & $28,475(61.1)$ & $1458(65.5)$ & *** \\
\hline \multicolumn{7}{|l|}{ Discharge facility type } \\
\hline Home self-care & $12,908(32.0)$ & $959(22.5)$ & $* * *$ & $9281(19.9)$ & $385(17.3)$ & *** \\
\hline $\mathrm{HHC}$ & $13,441(33.3)$ & $1606(37.8)$ & & $18,323(39.3)$ & $893(40.1)$ & \\
\hline SNF & $5185(12.8)$ & $652(15.3)$ & & $7008(15.0)$ & $357(16.0)$ & \\
\hline IRF & $8860(21.9)$ & $1036(24.4)$ & & $12,007(25.8)$ & $592(26.6)$ & \\
\hline \multicolumn{7}{|l|}{ Metro area } \\
\hline Metro & 37,794 (93.6) & $4209(99.0)$ & & $43,189(92.6)$ & $2201(98.8)$ & \\
\hline Nonmetro & $2600(6.44)$ & $44(1.03)$ & & $3430(7.36)$ & $26(1.17)$ & \\
\hline \multicolumn{7}{|l|}{ Insurance } \\
\hline Unknown/uninsured & $372(0.92)$ & $17(0.40)$ & $* * *$ & $226(0.48)$ & $11(0.49)$ & *** \\
\hline Medicare & $4262(10.6)$ & $858(20.2)$ & & $40,884(87.7)$ & 1909 (85.7) & \\
\hline Medicaid & $2838(7.03)$ & $1191(28.0)$ & & $63(0.14)$ & $34(1.53)$ & \\
\hline Commercial & $32,453(80.3)$ & $2142(50.4)$ & & $5364(11.5)$ & 259 (11.6) & \\
\hline Government & 469 (1.16) & $45(1.06)$ & & $82(0.18)$ & $14(0.63)$ & \\
\hline \multicolumn{7}{|l|}{ Volume of cases (by facility and year) } \\
\hline$<100 /$ year & $5803(14.4)$ & $888(20.9)$ & & $7438(16.0)$ & $424(19.0)$ & \\
\hline 100-199/year & $9621(23.8)$ & $641(15.1)$ & & $12,235(26.2)$ & $333(15.0)$ & \\
\hline $200+/$ year & $24,970(61.8)$ & $2724(64.0)$ & & $26,946(57.8)$ & $1470(66.0)$ & \\
\hline 90-day readmission & 3141 (7.78) & $510(12.0)$ & $* * *$ & $5198(11.1)$ & $292(13.1)$ & \\
\hline 90-day mortality & $50(0.12)$ & $13(0.31)$ & $* *$ & $312(0.67)$ & $8(0.36)$ & \\
\hline \multicolumn{7}{|l|}{ Elixhauser Index } \\
\hline 0 & 7304 (18.1) & $732(17.2)$ & & $8006(17.2)$ & $410(18.4)$ & \\
\hline $1-4$ & $30,492(75.5)$ & $3264(76.7)$ & & $35,726(76.6)$ & $1697(76.2)$ & \\
\hline$\geq 5$ & $2598(6.43)$ & $257(6.04)$ & & 2887 (6.19) & $120(5.39)$ & \\
\hline \multicolumn{7}{|l|}{ Surgery complications } \\
\hline Myocardial infarction & $22(0.05)$ & $1(0.02)$ & & $126(0.27)$ & $5(0.22)$ & \\
\hline Prosthetic device complication & $65(0.16)$ & $9(0.21)$ & & $98(0.21)$ & $4(0.18)$ & \\
\hline Surgical wound infection & $17(0.04)$ & $1(0.02)$ & & $17(0.04)$ & $0(0.00)$ & \\
\hline Venous thromboembolism & $11(0.03)$ & $2(0.05)$ & & $64(0.14)$ & $3(0.13)$ & \\
\hline
\end{tabular}

${ }^{a}$ Variables are compared by race for each age group (years $<65, \geq 65$ ) using Wald $\chi^{2}$ test from unadjusted binary or multinomial logistic regression models that account for clustering by facility. Significance levels: ${ }^{* *} p<0.01,{ }^{* * *} p<0.001$.

${ }^{\mathrm{b}} \mathrm{Clinical}$ comorbidities were identified based on coding algorithms developed by Quan et al. (enhanced Elixhauser version), using either the ICD-9CM or the ICD-10 coding system, as appropriate. The Elixhauser comorbidity index score is calculated based on the cumulative number of comorbidity conditions.

AA, African American; HHC, home health care; IRF, inpatient rehabilitation facility; SNF, skilled nursing facility; WH, White.

Similarly, in the older age group, compared with Home, discharge to IRF, SNF, or HHC, was associated with higher odds of 90-day readmission to acute care hospital (aOR: 1.70, 95\% CI: 1.53-1.88; aOR: 1.67, 95\% CI: 1.49-1.87; and aOR: $1.67,95 \%$ CI: 1.51-1.84, respectively). This association remained statistically significant after we repeated the analysis using CEM. The adjusted odds for IRF, SNF, and HHC were aOR: 1.61, 95\% CI: 1.43-1.82; aOR: 1.54, 95\% CI: $1.35-$ 1.77; and aOR: $1.54,95 \%$ CI: $1.38-1.73$, respectively.

\section{Discussion}

In this study of a large-scale regional database, we observed that there were significant differences between racial groups in postoperative discharge destination after elective THA. In this statewide dataset, when compared with whites, AAs were significantly more likely discharged to an Institution (IRFs or SNFs) or have HHC for postoperative care rather than home. We found that these differences persisted even after controlling for individual-level demographics, comorbidities, postoperative complications as well as facilitylevel characteristics. Furthermore, compared with home, discharge to IRF, SNF, and HHC was associated with higher odds of 90-day admission rate, even after adjusting for confounders and methodically matching of postop complications and comorbidities.

Earlier studies examining discharge destination after THA did not test the association of race. For example, in a multicenter study of patients from patients in Mayo clinic, Rochester; Massachusetts General Hospital, Boston, and University of California, San Francisco (4485 patients), 29\% were discharged to inpatient extended care facilities. Older age $>80$ or greater, female gender, higher comorbidities (American Society of Anesthesiologists class), and Medicare insurance 
Table 2. Characteristics by Discharge Locations of Total Hip Replacement Recipients $(N=93,493)$

\begin{tabular}{|c|c|c|c|c|c|}
\hline Variable, $n(\%)$ & Home self-care $(n=23,533)$ & $\mathrm{HHC}(n=34,263)$ & SNF $(n=13,202)$ & IRF $(n=22,495)$ & $p^{\mathrm{a}}$ \\
\hline \multicolumn{6}{|l|}{ Race } \\
\hline White & $22,189(94.3)$ & $31,764(92.7)$ & $12,193(92.4)$ & $20,867(92.8)$ & $* * *$ \\
\hline AA & $1344(5.71)$ & 2499 (7.29) & 1009 (7.64) & $1628(7.24)$ & \\
\hline \multicolumn{6}{|l|}{ Sex } \\
\hline Female & $11,296(48.0)$ & $19,343(56.5)$ & $7480(56.7)$ & $12,751(56.7)$ & $* * *$ \\
\hline Male & $12,237(52.0)$ & $14,920(43.5)$ & $5722(43.3)$ & $9744(43.3)$ & \\
\hline \multicolumn{6}{|l|}{ Age group } \\
\hline Years $<65$ & $13,867(58.9)$ & $15,047(43.9)$ & $5837(44.2)$ & $9896(44.0)$ & $* * *$ \\
\hline Years $\geq 65$ & $9666(41.1)$ & $19216(56.1)$ & 7365 (55.8) & $12,599(56.0)$ & \\
\hline \multicolumn{6}{|l|}{ Metro area } \\
\hline Metro & $21,124(89.8)$ & $32,458(94.7)$ & $12,507(94.7)$ & $21,304(94.7)$ & \\
\hline Nonmetro & 2409 (10.2) & 1805 (5.27) & $695(5.26)$ & 1191 (5.29) & \\
\hline \multicolumn{6}{|l|}{ Insurance } \\
\hline Unknown/uninsured & $225(0.96)$ & $207(0.60)$ & $76(0.58)$ & $118(0.52)$ & **** \\
\hline Medicare & $8961(38.1)$ & $19,092(55.7)$ & $7324(55.5)$ & $12,536(55.7)$ & \\
\hline Medicaid & $926(3.93)$ & $1575(4.60)$ & 629 (4.76) & $996(4.43)$ & \\
\hline Commercial & $13,223(56.2)$ & $13,203(38.5)$ & 5085 (38.5) & 8707 (38.7) & \\
\hline Government & $198(0.84)$ & $186(0.54)$ & $88(0.67)$ & $138(0.61)$ & \\
\hline \multicolumn{6}{|l|}{ Volume of cases (by facility and year) } \\
\hline$<100 /$ year & $2231(9.48)$ & 5976 (17.4) & $2378(18.0)$ & 3968 (17.6) & \\
\hline 100-199/year & $4155(17.7)$ & $9254(27.0)$ & $3313(25.1)$ & $6108(27.2)$ & \\
\hline $200+/$ year & $17,147(72.9)$ & $19,033(55.5)$ & $7511(56.9)$ & $12,419(55.2)$ & \\
\hline 90-day readmission & $1476(6.27)$ & $3766(11.0)$ & $1424(10.8)$ & $2475(11.0)$ & $* * *$ \\
\hline 90-day mortality & $25(0.11)$ & $184(0.54)$ & $63(0.48)$ & $111(0.49)$ & $* * *$ \\
\hline \multicolumn{6}{|l|}{ Elixhauser Index } \\
\hline 0 & $4203(17.9)$ & $6006(17.5)$ & 2317 (17.6) & 3926 (17.5) & \\
\hline $1-4$ & $17,852(75.9)$ & $26,096(76.2)$ & $10,061(76.2)$ & $17,170(76.3)$ & \\
\hline$\geq 5$ & $1478(6.28)$ & $2161(6.31)$ & $824(6.24)$ & $1399(6.22)$ & \\
\hline \multicolumn{6}{|l|}{ Surgery complications } \\
\hline Myocardial infarction & $12(0.05)$ & $69(0.20)$ & $28(0.21)$ & $45(0.20)$ & $* * *$ \\
\hline Prosthetic device complication & $21(0.09)$ & $81(0.24)$ & $28(0.21)$ & $46(0.20)$ & $* * *$ \\
\hline Surgical wound infection & $7(0.03)$ & $18(0.05)$ & $5(0.04)$ & $5(0.02)$ & \\
\hline Venous thromboembolism & $3(0.01)$ & $48(0.14)$ & $14(0.11)$ & $15(0.07)$ & $* * *$ \\
\hline
\end{tabular}

aVariables are compared by discharge destination using Wald $\chi^{2}$ test from unadjusted binary or multinomial logistic regression models that account for clustering by facility. Significance levels: ${ }^{* * *} p<0.001$

${ }^{b}$ Clinical comorbidities were identified based on coding algorithms developed by Quan et al. (enhanced Elixhauser version), using either the ICD-9CM or the ICD-10 coding system, as appropriate. The Elixhauser comorbidity index score is calculated based on the cumulative number of comorbidity conditions.

were most significant predicators of discharge to an inpatient facility, however, patient race was not reported in this study. ${ }^{22}$ However, our findings are similar to the previous studies that have examined race. ${ }^{23,24}$ In a single institutional administrative data study, minorities were more likely to be assigned to an institution after discharge (OR: 2.11). ${ }^{25}$ In a larger study using California statewide data $(n=14,326)$, race, insurance, and morbidity were the main driving factors on patient discharge to $\mathrm{SNF}^{26}$

With regard to readmission, a single-center study from New York using hospital records from 2010 to 2011 demonstrated that patients discharged home with health services has significantly lower readmission rates than those discharged to IRFs (1.5\% vs. $5.1 \%) .{ }^{27} \mathrm{~A}$ study from Kaiser Permanente total joint replacement registry from 2001 to $2004\left(\mathrm{n}_{\mathrm{THA}}=3432\right)$, after controlling for the comorbidities, age, sex, and hospital complications, the records of hospital readmission were higher in patients discharge to an $\mathrm{SNF}^{28}$ Finally, in a previous study by our team analyzing the PHC4 database from 2002 to 2012, similarly demonstrated that when compared with whites, AA patients had significantly higher odds of discharge to IRF (age $<65$, aRRR =2.56; age $\geq 65, a R R R=1.96$ ) and to SNF (age $<65$, aRRR $=3.37$; age $\geq 65$, aRRR $=3.66$ ). In addition, the OR of 90-day readmission was higher in patients who were discharged to IRF or SNF irrespective of the age group (age $<65, \mathrm{OR}_{\mathrm{IRF}} 4.06, \mathrm{OR}_{\mathrm{SNF}} 2.05$; age $\left.\geq 65, \mathrm{OR}_{\mathrm{IRF}} 4.32, \mathrm{OR}_{\mathrm{SNF}} 1.74\right) .^{12}$

Our results demonstrating that AA patients are more likely than white patients to be discharged to an institution (IRF/SNF) or HHC for postoperative care following elective THA are important. We demonstrated that even after the change in CMS policies, marked disparities remain in our analysis from 2012 to 2016. However, it is important to note that the aRRRs have improved for AAs to be discharged to a 


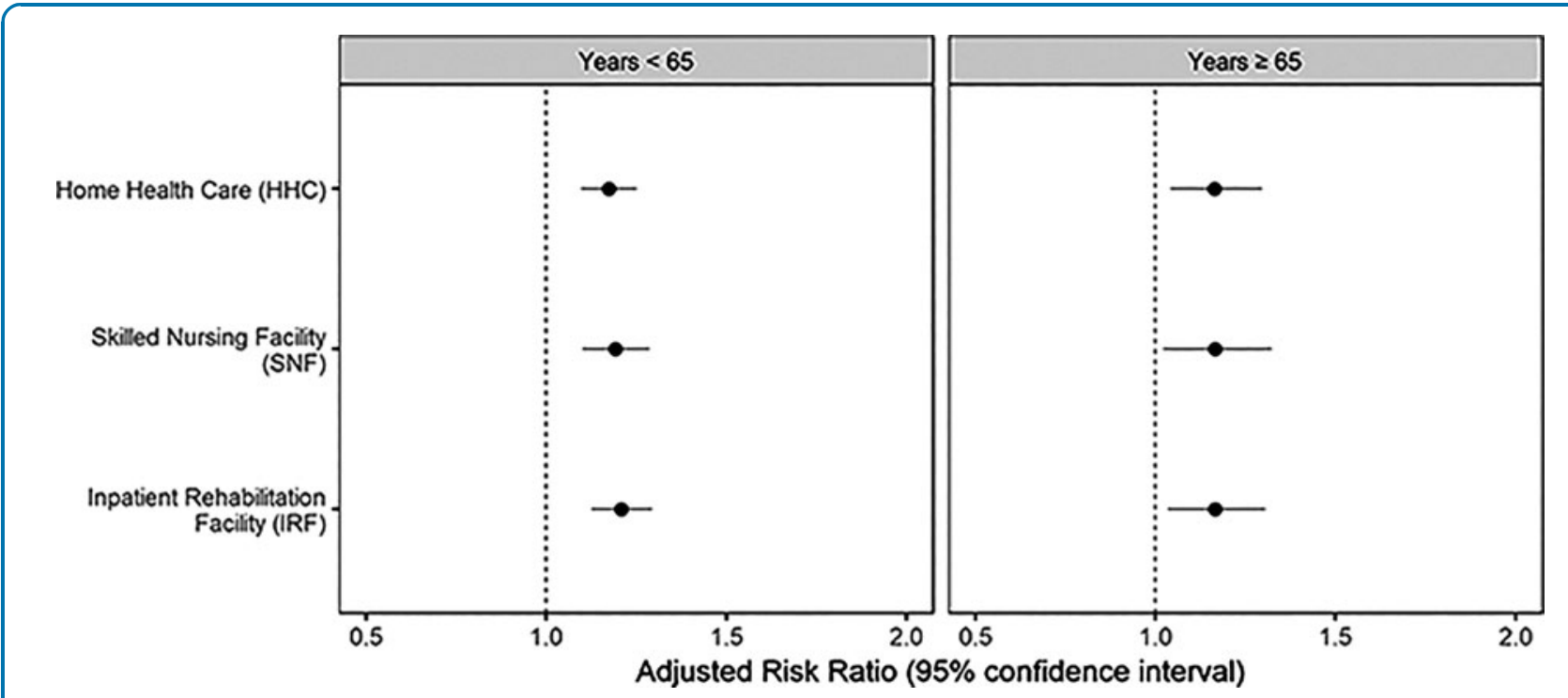

FIG. 2. Adjusted relative risk ratios of referral to varying discharge locations in African American THA patients (vs. whites) in two age groups. The generalized multinomial logit model accounts for clustering by facility. Adjusted covariates included patient sex, insurance, facility type, facility volume of cases, Elixhauser Index, and surgical complications (venous thromboembolism, prosthetic device complications, myocardial infarction, and wound infections). THA, total hip arthroplasty.

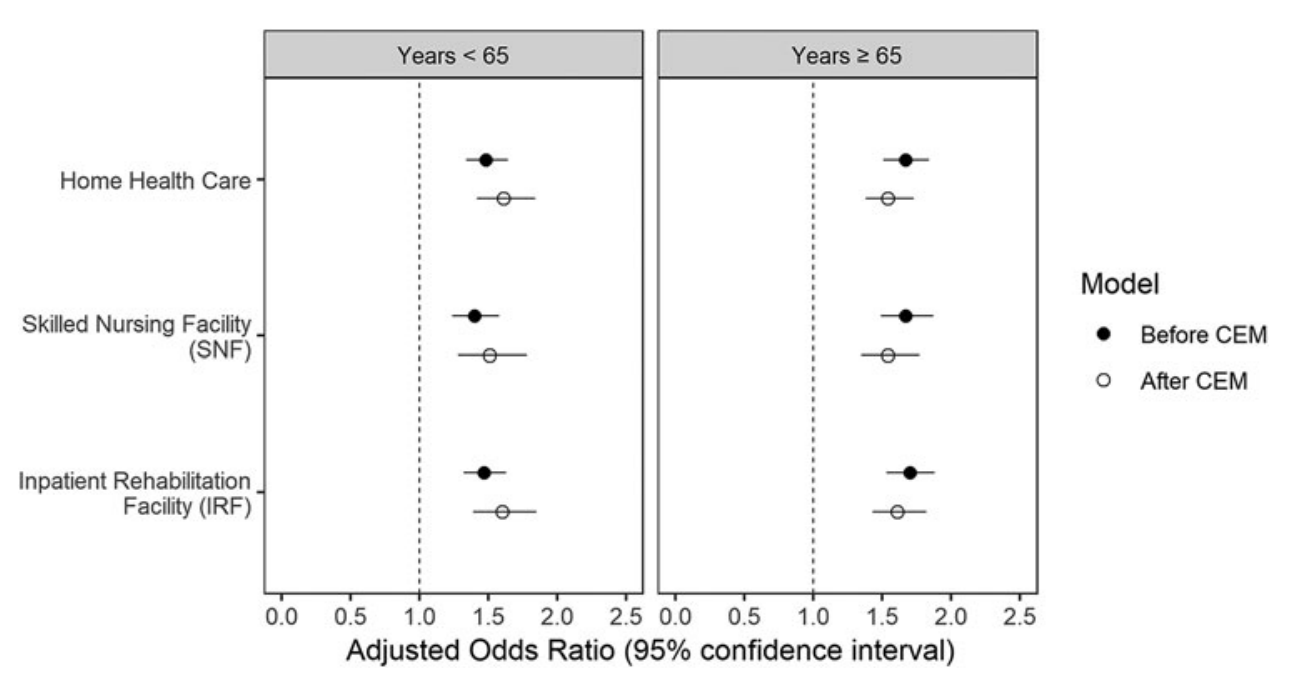

FIG. 3. Adjusted odds ratios of 90-day readmission in patients who were discharged to various locations compared with home before and after CEM. The binary logistic 90-day readmission models adjusted for patient sex, race, insurance, Elixhauser Index, facility type, facility volume of cases, and surgical complications. CEM, coarsened exact matching. 
nonhome disposition. In addition, the ORs for 90-day readmission to an acute care hospital have decreased. The CEM method confirmed the findings of the adjusted model. However, patients $<65$ years had a stronger predisposition, whereas patients $>65$ years had a weaker predisposition to 90-day readmission after CEM. Thus, describing that the matching made a difference in effect sizes, however, the association remained significant. Studies have shown that early discharge to home is associated with reduced cost, improved clinical outcomes, and increased patient satisfaction. ${ }^{29,30}$ Thus, our study findings are significant since elective THA is one of the most important cost centers for insurances and postoperative care contributed a significant amount to the costs. ${ }^{29-31}$ Also, discharge to an institution is associated with higher odds of readmission rates, which is a key outcome measure post THA. ${ }^{32}$ It is possible that the discharge destination is not only determined by clinical parameters but also some social determinants like proximity to home, social and community support, or overall deprivation or social vulnerability, of which race may be a marker. ${ }^{32,33}$

Our study has several limitations. This large administrative database does not contain information on potentially important confounding variables such as BMI, social support or patient preference in disposition, or regarding patient-reported outcomes, and we are unable to comment on these factors. Several patients may have transferred between different facilities during postoperative care and it is difficult to capture these adequately and thus, the crossover effect cannot be estimated in our study. We studied only patients with primary elective THA in the Pennsylvania, thus, our results may not be generalizable to other regions or states of the country, because there is a significant geographical variation in several key factors, including social determinants of health. ${ }^{34}$

\section{Conclusion}

In summary, this large-scale study of 99,171 patients undergoing primary THA across 170 Pennsylvania hospitals demonstrated that as compared with whites, AA patients were more likely to be referred to institution (IRFs and SNFs) or HHC for postoperative care rather than home. Moreover, discharge to an institution or HHC for postoperative care increases the odds of 90-day hospital readmission when compared with being discharged home. Evaluations of the decision process regarding discharge destination for posta- cute care and rehabilitation following elective THA are needed. We also need studies to examine how social determinants of health such as patient race impact these decisions.

\section{Author Disclosure Statement}

No competing financial interests exist

\section{Funding Information}

S.A.I. is supported by grant number K24AR055259 from the National Institute of Arthritis and Musculoskeletal and Skin Diseases. B.M. is supported by the C. Ronald MacKenzie Young Scientist Endowment Award.

\section{References}

1. Murray CJ, Atkinson C, Bhalla K, et al. The state of US health, 1990-2010: burden of diseases, injuries, and risk factors. JAMA. 2013;310: 591-608.

2. Wallace IJ, Worthington S, Felson DT, et al. Knee osteoarthritis has doubled in prevalence since the mid-20th century. Proc Natl Acad Sci U S A. 2017;114:9332-9336.

3. HCUP. Available at https://www.hcup-us.ahrq.gov/reports/statbriefs/ sb186-Operating-Room-Procedures-United-States-2012.jsp Accessed December 26, 2018.

4. Pabinger $C$, Geissler A. Utilization rates of hip arthroplasty in OECD countries. Osteoarthritis Cartilage. 2014;22:734-741.

5. Wolford M, Palso K, Bercovitz A. Hospitalization for total hip replacement among inpatients aged 45 and over: United States. NCHS Data Brief. 2000;2010:1-8.

6. Kurtz S, Ong K, Lau E, et al. Projections of primary and revision hip and knee arthroplasty in the United States from 2005 to 2030. JBJS. 2007;89:780-785.

7. Jordan JM, Linder GF, Renner JB, et al. The impact of arthritis in rural populations. Arthritis Care Res. 1995;8:242-250.

8. Jha AK, Fisher ES, Li Z, et al. Racial trends in the use of major procedures among the elderly. N Engl J Med. 2005;353:9.

9. Mahomed NN, Barrett JA, Katz JN, et al. Rates and outcomes of primary and revision total hip replacement in the United States medicare population. J Bone Joint Surg Am. 2003;85-a:27-32.

10. Weaver F, Hynes D, Hopkinson W, et al. Preoperative risks and outcomes of hip and knee arthroplasty in the Veterans Health Administration. J Arthroplasty. 2003;18:693-708.

11. Lavernia CJ, Alcerro JC, Contreras JS, et al. Ethnic and racial factors influencing well-being, perceived pain, and physical function after primary total joint arthroplasty. Clin Orthop Relat Res. 2011;469: 1838-1845.

12. Vina ER, Kallan MJ, Collier A, et al. Race and rehabilitation destination after elective total hip arthroplasty: analysis of a large regional data set. Geriatr Orthop Surg Rehabil. 2017;8:192-201.

13. Bundled Payments for Care Improvement (BPCI) Initiative: General Information. Available at https://innovation.cms.gov/initiatives/ bundled-payments Accessed December 26, 2018.

14. Singh J, Kwoh C, Boudreau R, et al. Hospital volume and surgical outcomes after elective hip/knee arthroplasty: a risk-adjusted analysis of a large regional database. Arthritis Rheum. 2011;63:2531-2539.

15. Rural-Urban Continuum Codes. 2013. Available at www.ers.usda.gov/ data-products/rural-urban-continuum-codes/documentation.aspx Accessed June 13, 2016.

16. (HCUP) AHCaUP. HCUP Elixhauser Comorbidity Software. 2016. Available at www.hcup-us.ahrq.gov/toolssoftware/comorbidity/ comorbidity.jsp Accessed November 14, 2019.

17. Quan $H$, Sundararajan V, Halfon $P$, et al. Coding algorithms for defining comorbidities in ICD-9-CM and ICD-10 administrative data. Med Care. 2005;43:1130-1139. 
18. Tom A, Bosker TASRJ, Bosker RJ. Multilevel Analysis: An Introduction to Basic and Advanced Multilevel Modeling. Sage, 1999.

19. lacus SM, King G, Porro G. Causal inference without balance checking: coarsened exact matching. Political Anal. 2012;20:1-24.

20. Ho DE, Imai K, King G, et al. Matching as nonparametric preprocessing for reducing model dependence in parametric causal inference. Political Anal. 2007;15:199-236.

21. Ho DE, Imai $K$, King $G$, et al. Matchlt: nonparametric preprocessing for parametric causal inference. J Statistical Software. 2011;42:1-28.

22. Bozic KJ, Wagie A, Naessens JM, et al. Predictors of discharge to an inpatient extended care facility after total hip or knee arthroplasty. J Arthroplasty. 2006;21:151-156.

23. Inneh IA, Clair AJ, Slover JD, et al. Disparities in discharge destination after lower extremity joint arthroplasty: analysis of 7924 patients in an Urban setting. J Arthroplasty. 2016;31:2700-2704.

24. Morrow-Howell N, Proctor E. Discharge destinations of Medicare patients receiving discharge planning: who goes where? Medical Care. 1994;32: 486-497.

25. Lan $\mathrm{RH}$, Kamath AF. Post-acute care disparities in total joint arthroplasty. Arthroplast Today. 2017;3:187-191.

26. Schwarzkopf R, Ho J, Snir N, et al. Factors influencing discharge destination after total hip arthroplasty: a California state database analysis. Geriatr Orthop Surg Rehabil. 2015;6:215-219.

27. Ramos NL, Karia RJ, Hutzler LH, et al. The effect of discharge disposition on 30-day readmission rates after total joint arthroplasty. J Arthroplasty. 2014;29:674-677.

28. Bini SA, Fithian DC, Paxton LW, et al. Does discharge disposition after primary total joint arthroplasty affect readmission rates? J Arthroplasty. 2010;25:114-117.

29. Bozic KJ, Ward L, Vail TP, et al. Bundled payments in total joint arthroplasty: targeting opportunities for quality improvement and cost reduction. Clin Orthop Relat Res. 2014;472:188-193.

30. Bundled Payments for Care Improvement (BPCI). Initiative: general information. Available at http://innovation.cms.gov/initiatives/bundledpayments/index.html Accessed November 14, 2019.
31. London DA, Vilensky S, O'Rourke C, et al. Discharge disposition after joint replacement and the potential for cost savings: effect of hospital policies and surgeons. J Arthroplasty. 2016;31:743-748.

32. de Pablo $P$, Losina $E$, Phillips $C B$, et al. Determinants of discharge destination following elective total hip replacement. Arthritis Rheum. 2004:51:1009-1017.

33. Oldmeadow LB, McBurney $\mathrm{H}$, Robertson VJ. Predicting risk of extended inpatient rehabilitation after hip or knee arthroplasty. J Arthroplasty. 2003;18:775-779.

34. Bustamante-Zamora D, Maizlish N. Cross-sectional analysis of two social determinants of health in California cities: racial/ethnic and geographic disparities. BMJ Open. 2017;7:e013975.

Cite this article as: Mehta B, Singh JA, Ho K, Parks M, Nelson C, D'Angelo D, Ibrahim SA (2019) Race, discharge disposition, and readmissions after elective hip replacement: analysis of a large regional dataset, Health Equity 3:1, 628-636, DOI: 10.1089/heq.2019.0083.

\section{Appendix Table A1. ICD-9/10 Codes of Inclusion Hip Procedure and Surgical Complications}

\begin{tabular}{|c|c|c|}
\hline & ICD 9 codes & ICD 10 codes \\
\hline Hip replacement & 8151 & OSR90xx, OSRB0xx \\
\hline Venous thromboembolism & $\begin{array}{l}45340,45341,4536,45342,45384,45381 \\
\quad 45386,45389,45382,4539,45385\end{array}$ & $\begin{array}{l}182441,182433,182 B 19,182409,182621,182411,182402,182401, \\
\quad \text { I824Z9, I82491, I824Z1, I82432, I82412, I824Z2, I82512 }\end{array}$ \\
\hline Acute myocardial infarction & $\begin{array}{l}41071,41021,41011,41072,41081,41001 \\
\quad 41061,41091,41042,41070,41090,41020 \\
\quad 41041\end{array}$ & $1214,1213,12129,12119,12102$ \\
\hline Surgical wound infection & 99859 & K6811, T814XXA \\
\hline Prosthetic device complications & $\begin{array}{l}99677,99647,99642,99678,99644,99640 \\
\text { 99649, } 99643,99666,99667,99641,99601 \\
\text { 99646, } 99679\end{array}$ & $\begin{array}{l}\text { T8481XA, T84115D, T84020A,T84041A, T84114A, T84218A, } \\
\text { T8484XA, T84498A, T84199A, T84011A, T84021A, T8489XA, } \\
\text { T8451XA, T84010A, T84091A, T84031A, T84328A, T84030A, } \\
\text { T847XXA, T84050S, T84099A, T84040A, T84090A, T84428A, } \\
\text { T8451XD, T84038A, T84061A, T84050A, T8486XA, T8452XA, } \\
\text { T8483XA }\end{array}$ \\
\hline
\end{tabular}

